\title{
Structural and Elemental Analysis on Bismuth Ferrite-Doped Ceramics: An Energy Dispersive Spectroscopy and Rietveld Analysis
}

\author{
G. Rojas-George ${ }^{1}$, G. Herrera-Perez ${ }^{1}$, H. E. Esparza-Ponce ${ }^{2}$, A. Reyes-Rojas ${ }^{2}$ \\ ${ }^{1}$ CONACyT Research Fellow, Centro de investigación en Materiales Avanzados, S.C. (CIMAV), \\ Miguel de Cervantes 120, Complejo Industrial Chihuahua, Chihuahua, México. \\ ${ }^{2}$ Department of Physics of Materials, Centro de investigación en Materiales Avanzados, S.C. (CIMAV), \\ Miguel de Cervantes 120, Complejo Industrial Chihuahua, Chihuahua, México.
}

Monophasic multiferroic materials have attracted great interest lately; in consequence extensive research has been performed on these multifunctional ceramics. Bismuth Ferrite (BFO), a monophasic multiferroic oxide possess a rombohedral distorted perovskite structure, exhibits, both ferroelectricity and weak antiferromagnetism (spin cycloid of $64 \mathrm{~nm}$ ) at room temperature [1]. These characteristics make this ceramic suitable for a great variety of electronic applications, such as spintronic applications. BFO possess multifunctional qualities that no other material possess, however obtaining this material in pure phase is quite an accomplishment. Along with the hardships of suppressing the spin cycloid and most important suppressing the current leak associated with this material. The current leak is usually associated to oxygen vacancies; however, the stoichiometric relation between bismuth and iron ions is not quite reported in the literature. This could also be a factor for the lack of outstanding multiferroic properties.

Titanium-barium-cobalt doped bismuth ferrite nanoparticles $\left(\mathrm{Bi}_{0.95} \mathrm{Ba}_{0.05} \mathrm{Fe}_{0.98} \mathrm{Co}_{0.01} \mathrm{Ti}_{0.01} \mathrm{O}_{3}\right)$ were synthesized by a modified Pechini method [2]. Special care was taken during the synthesis process, since excesses of 4,5 , and $6 \%$ of bismuth was used during the preparation of the ceramic powders, to compensate bismuth loss and to obtain a 1:1 stoichiometric relation between bismuth and iron. Ceramic pellets were obtained after different sintering temperatures $\left(710,800,850{ }^{\circ} \mathrm{C}\right)$, times $(2 \mathrm{~min}, 1,2,4,6$ and $8 \mathrm{~h}$ ) and techniques were implemented. The powders and ceramic samples were characterized through X-ray diffraction (XRD) to confirm the phase, scanning electron microscopy - Energy dispersive spectroscopy (SEM-EDS) was used to quantify the atomic relation between the cations. Rietveld refinement was done to clarify the structure.

The excess used during the synthesis produces modifications in the final crystal structure, phase purity, crystal size and particle size on powders and ceramic samples. XRD and Rietveld refinements revealed that using $4 \%$ excess of bismuth during the synthesis and a heat treatment of $600{ }^{\circ} \mathrm{C}$ for $2 \mathrm{~h}$ on the powders and using the rapid liquid phase sintering technique at $790{ }^{\circ} \mathrm{C}$ for 2 minutes, a pure phase of BFO (PDF 01-077-5211) sample is obtained.

Scanning Electron Microscopy shows a particle size around $100 \mathrm{~nm}$ of acicular shape (fig 1a), for the ceramic sintered at $790{ }^{\circ} \mathrm{C}$ for 2 minutes. This behavior is attributed to the liquid phase formed during the sintering; since this technique takes only seconds, nucleation and phase formation is rapid, allowing the formation of crystals only on the surface of the ceramic. In this way, nucleation increases reducing crystal growth rate. Performing an Energy Dispersive Spectroscopy analysis, it was found that using 4\% of excess during the synthesis a relation of $\mathrm{Bi}: \mathrm{Fe}$, including the corresponding dopants, a stoichiometric relation of 1:1 was reached( Fig 1b). 
Rietveld refinement (fig 2) confirms the incorporation of all dopants ( $\mathrm{Ti}, \mathrm{Ba}, \mathrm{Co}$ ). The profile was carried out considering an $R 3 c$ symmetry which is in good agreement with the regular rombohedral structure. Rietveld results show a small difference between the experimental and calculated pattern; obtaining a $\chi^{2}$ of 1.3 in the Rietveld fitting, with crystallographic parameters of $\mathrm{a}_{\text {rhom }}=5.5921(1) \AA$, and $\mathrm{c}_{\text {rhom }}=$ 13.8781(1) $\AA$. The bond lengths were $\mathrm{Fe}_{-} \mathrm{O}_{(1)}=1.94 \AA$ and $\mathrm{Fe}-\mathrm{O}_{(2)}=2.12 \AA$, length ratio was $\mathrm{Fe}-$ $\mathrm{O}_{(1)} / \mathrm{Fe}-\mathrm{O}_{(2)}=0.91$, and bond angle was $\mathrm{Fe}-\mathrm{O}-\mathrm{Fe}=156.2^{\circ}$. Therefore, from these calculated results and from the length ratio $\mathrm{Fe}-\mathrm{O}_{(1)} / \mathrm{Fe}-\mathrm{O}_{(2)}=0.92\left(\mathrm{BiFeO}_{3}\right)$ it is possible to infer a degree of $1.1 \%$ rhombohedrally-distorted perovskite compared to undoped $\mathrm{BiFeO}_{3}$.

In summary, the EDS analysis of the sample synthesized with $4 \%$ excess of bismuth and sintered at 790 ${ }^{\circ} \mathrm{C}$ for 2 minutes gives a stoichiometric relation of $\mathrm{Bi}: \mathrm{Fe}$ of $1: 1$. Rietveld analysis confirms the $R 3 c$ symmetry and rombohedral structure in the sample [3].

\section{References:}

[1] G.A. Smolenskii et al, Sov. Phys. Solid State 6 (1965) p. 2936.

[2] G. Rojas-George et al Mat. Chem Phys 146 (2014) p. 73.

[3] The authors acknowledge the contributions done by David Moya Jurado and Karla Campos Venegas.
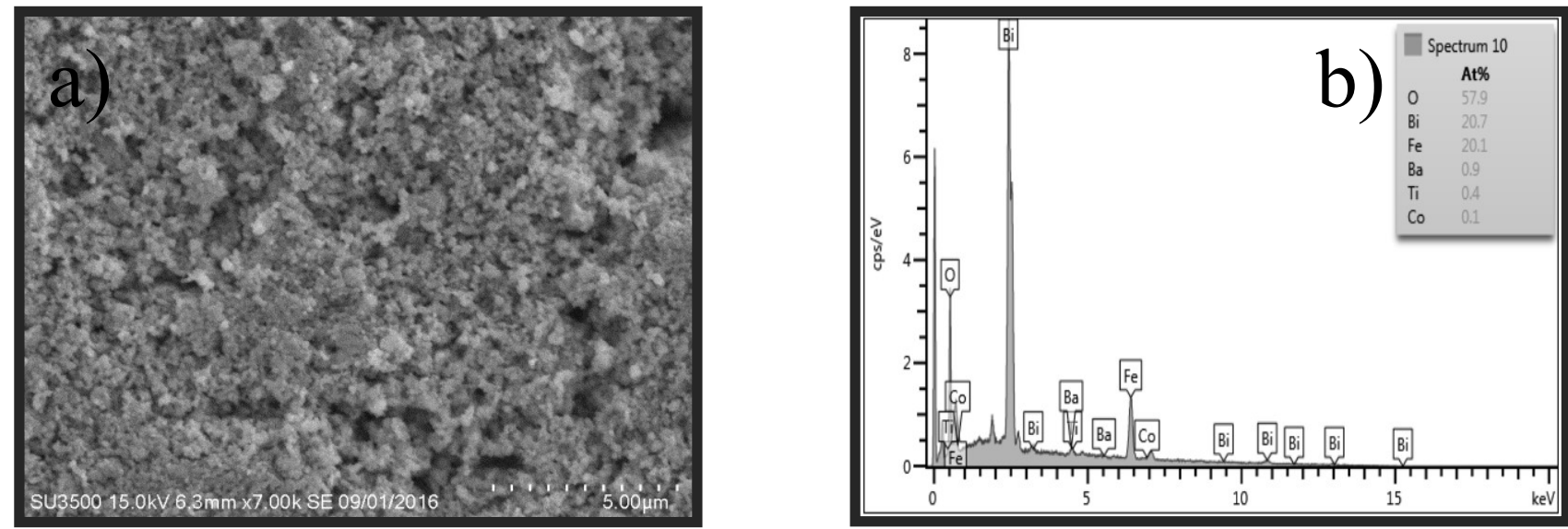

Figure 1. a) SEM image and b) EDS spectrum of $\mathrm{Bi}_{0.95} \mathrm{Ba}_{0.05} \mathrm{Fe}_{0.98} \mathrm{Co}_{0.01} \mathrm{Ti}_{0.01} \mathrm{O}_{3}$ ceramic sintered at $790{ }^{\circ} \mathrm{C}$ for 2 min synthesized with $4 \%$ excess of bismuth.

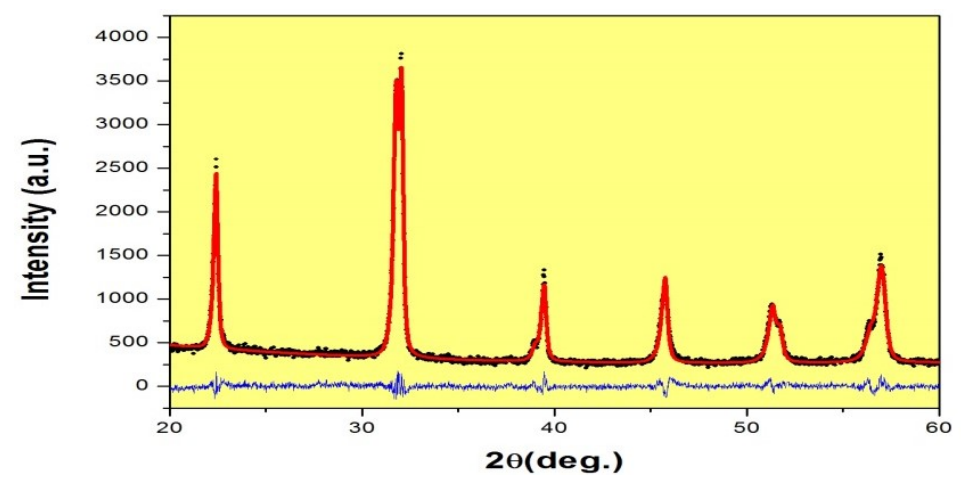

Figure 2. Rietveld refinement of $\mathrm{Bi}_{0.95} \mathrm{Ba}_{0.05} \mathrm{Fe}_{0.98} \mathrm{Co}_{0.01} \mathrm{Ti}_{0.01} \mathrm{O}_{3}$ ceramic sintered at $790{ }^{\circ} \mathrm{C}$ for 2 min synthesized with $4 \%$ excess of bismuth. The difference between observed and calculated pattern intensity is shown at the bottom of the pattern 